\title{
INTEGRATED COMPUTER SYSTEMS. ENTERPRISE RESOURCE PLANNING (ERP)
}

\author{
Dan Mircea TRANĂ, Assoc. prof. Ph.D. \\ Faculty of Accounting and Finance, Râmnicu Vâlcea \\ Spiru Haret University \\ dan.trana@spiruharet.ro
}

\begin{abstract}
At the beginning of the XXI century society, knowledge based society, the management of economic organizations can only be achieved through optimal IT systems. They can be seen as an extension of increasingly complex information systems and provide effective leadership only if they are integrated in the economic system of the organization. We have previously shown some of the features that recommend integrated IT systems to be controlled and used, as well as main principles for building the integrated computer systems, strategies that can be applied in the designing of this type of IT system. Advantages of management integrated IT systems can be best supported by examples, and therefore we intend to present a special category, but increasingly used, of integrated IT systems: Enterprise Resource Planning (ERP). They are "distributed IT systems based on client / server and developed for the processing of transactions and facilitating the integration of business processes with suppliers, customers and other business partners."
\end{abstract} planning

Keywords: system, intelligent technology (IT), knowledge, integrated,

JEL Classification: $\mathrm{C}_{8}, \mathrm{C}_{88}$

\section{Introduction}

ERP is short for Enterprise Resource Planning. It is a business process management software that allows an organization to use a system of integrated applications to manage the business and automate back office functions. ERP software integrates all facets of an operation, including product planning, development, manufacturing processes, sales and marketing.

ERP Software Modules consists of multiple enterprise software modules that are individually purchased, based on what best meets the specific needs and technical capabilities of the organization. Each ERP module is focused on one area of business processes, such as product development or marketing. A business scan use ERP software to manage back-office activities and tasks including the following: distribution process management, supply chain management, services knowledge base, configure, prices, improve accuracy of financial data, facilitate better project planning, automate employee life-cycle, standardize critical business 
procedures, reduce redundant tasks, assess business needs, accounting and financial applications, lower purchasing costs, manage human resources and payroll.

Some of the most common ERP modules include those for product planning, material purchasing, inventory control, distribution, accounting, marketing, finance and HR.

As the ERP methodology has become more popular, software applications have emerged to help business managers implement ERP into other business activities and may also incorporate modules for CRM and business intelligence and present them as a single unified package.

The basic goal is to provide one central repository for all information that is shared by all the various ERP facets in order to smooth the flow of data across the organization.

\section{Theoretical background}

We make a brief and concise summary of the main elements in the evolution of IT systems in general and management in particular.

In the 1960s, intelligent technology focused on systems using independent data collections managed by programs written in advanced programming languages such as COBOL and FORTRAN. The applications are developed in-house for inventory management, personnel and payroll records, records of fixed assets, purchase and sale, general accounting.

The year 1965 marks the emergence and use of applications specially designed for programming and tracking production. Material Requirements Planning systems type (MRP) allows users to automatically determine the necessary products based on production schedule, inventory and data about stocks, information that could be made available to the manager by the help of already operating IT systems, in that period of time. Designed to coordinate production, they allow better timing between required materials and requirements of the production process. Consequently, there is an optimization of the supply activity, eliminating unnecessary inventories of raw materials and cutting down storage costs, eliminating or limiting production goal situations due to lack of raw material.

The concerns of designers for continuous improvement of the facilities provided by IT systems that support the leadership of economic systems are rewarded by the first Enterprise Resource Planning systems (ERP) developed in 1987. They extend the use of the systems in fields as manufacturing, financial, accounting, distribution of goods, supplies, human resources management, stocks of raw materials or finished products, office supplies and secretary area, technological design. It is thus a major step towards the designing of IT systems with relational databases and the integration of IT systems in the economic systems of increasingly complex organizations.

Since 1990, there is a continuous development of ERP systems, the designers focused on uncharted sectors of production and their economic development, building up systems such as Customer Relationship Management (CRM), Supply Chain Management (SCM), Advanced Planning and Scheduling (APS), Enterprise 
Integration Application (EIA), Business Process Integration (BPI). The tendency to develop highly integrated IT systems on existing economic systems becomes more and more obvious, even anticipating the future ones.

\section{General considerations [3]}

Coming into being around the year 1987, ERP IT systems are modular software packages for management activities of economic organizations. American Production and Inventory Control Society (APICS) define ERP IT systems as a "method for effective planning and control of resources for management of customer orders of an economic organization".

Regarded as integrated systems, ERP systems provide:

- the integration of functionalities;

- the integration of databases (with operation collection, storage, processing, distribution, communication) constituting data stores;

- the integration of basic and application programs.

An integrated ERP IT system has the following characteristics:

- the works on the architecture of client / server type;

- it is self-adaptable, performing automated tasks without the operator intervention;

- integrates and unifies business processes;

- process organization transactions;

- use a single database following the principle of minimum redundancy;

- data access can be done in real time;

- perfectly fit the specific industry that the economic organization is part of;

- provide multilingual and multi-currency support.

The use of integrated ERP systems have a number of advantages such as:

- high quality of information and knowledge provided;

- time response close to that of real-time work;

- minimum redundancy of data and operations;

- scalability;

- can be easily adapted to the activity of the organization;

- provides a framework for collaborative work;

- are e-business oriented.

Even though it is very modern and has many advantages, by the quick and easy integration in complex economic systems, ERP systems has some disadvantages:

- ERP projects are time consuming and increase the time response of the system;

- there are situations in which solutions offered by ERP modules are not exactly applicable to the user's intentions regarding the business processes and strategic objectives of the economic organization;

- ERP projects show a clear dependence on the supplier;

- ERP modules are highly complex and require operational developments during the working period of the ERP system life cycle. 
Finally, we present an integrated ERP package. Integrated organizational system contains components (on request) for all functions of a business organization performance.

Accounting management component provides integrated management of heritage, both financially as well as accounting and adapts to any type of organization, regardless of size, complexity of business areas and ownership structure. Financial and accounting records are complete at the analytic and synthetic level. Default accounting balances are updated after each accounting operations.

Supply management component provides the necessary supply operative setting, selection of the best price, the optimal quantities and conditions of supply by managing suppliers, procurement and analysis of their history, automatic track purchase requests, requests for proposals, tenders and orders, and quality while optimizing the entire supply activities.

Management Information System (MIS Business Analizer ensure monitoring and correlation of all levels of an organization of economic activity, enabling analytical and synthetic data processing in real time decision support and information. Through integration with Oracle Express product enables the use of OLAP technology.

The ERP field can be slow to change, but the last couple of years have unleashed forces, which are fundamentally shifting the entire area. According to Enterprise Apps Today, the following new and continuing trends affect enterprise ERP software:

\section{MOBILE ERP}

Mobility continues to be a big trend. Executives and employees want realtime access to information, regardless of where they are. It is expected that businesses will quickly embrace mobile ERP, not just for reports and dashboards, but for conducting key business processes.

\section{CLOUD ERP}

The cloud has been advancing steadily into the enterprise for some time, but many ERP users have been reluctant to place data cloud. Those reservations have gradually been evaporating, however, as the advantages of the cloud become apparent.

\section{SOCIAL ERP}

There has been much hype around social media and how important - or not -it is to add to ERP systems. Certainly, vendors have been quick to seize the initiative, adding social media packages to their ERP systems with much fanfare. But some wonder if there is really much gain to be had by integrating social media with ERP. 


\section{TWO-TIER ERP}

Enterprises once attempted to build an all-encompassing ERP system to take care of every aspect of organizational systems. But some expensive failures have gradually brought about a change in strategy - adopting two tiers of ERP.

\section{What is small business ERP?}

In ERP terminology, the phrase small business ERP is used to describe a lightweight business management software that is designed to meet the needs of a small business.

ERP software integrates all facets of an operation, including development, manufacturing, sales and marketing. Small business ERP is typically a SaaS (software as a service) model and includes project management, financials, manufacturing, warehouse management, accounting, sales and business management.

Typically, ERP software is considered an enterprise application and it is designed for larger enterprises that require dedicated teams to customize, analyse the data and reports and handle upgrades and deployment. In contrast, Small business ERP applications differ in a number of ways including the amount of data handled by the system and less-complex screens and dashboards. Support is offered by the provider and the software is customized for the business industry you work in.

\section{Application Service Providers (ASP)}

Today, using enterprise application service providers (ASP) is more prevalent. Here, the enterprise application is designed by a third-party application service provider and leased to the enterprise, as an on-premise or hosted service. This is also often referred to software-as-a-service (SaaS) or Web-based applications.

Another trend in enterprise applications is the move to cloud computing, where the enterprise moves some or its entire infrastructure to the cloud - a type of Internet-based computing, where services are delivered to an organization's computers and devices through the Internet as an on-demand service. Some enterprises may also choose a hybrid solution where cloud applications are integrated with on-premise systems.

Some of the more common types of enterprise applications include the following:

- automated billing systems

- payment processing

- email marketing systems

- content management

- call centre and customer support

- Customer Relationship Management (CRM)

- Enterprise Resource Planning (ERP)

- Business Intelligence 
- Business Continuity Planning (BCP)

- HR Management

- Enterprise Application Integration (EAI)

- enterprise search

- messaging and collaboration systems.

Other common names for enterprise application include enterprise app, enterprise software and enterprise application software (EAS).

\section{What is enterprise search?}

Enterprise search is an extensive search system that provides the means to search both structured and unstructured data sources with a single query. It addresses the needs of businesses that need to store, retrieve and track digital information of all kinds. Data sources in enterprise search systems includes information stored in many different containers such as e-mail servers, application databases, content management systems, file systems, intranet sites and external Web sites. Enterprise search systems provide users with fast query times and search results that are usually ranked in such a way that the information you need is easily accessible.

Enterprise search systems typically do not search the public Internet, but rather everything that is contained within the organization's own private corporate network, on the inside of the firewall.

\section{What is enterprise messaging system?}

Abbreviated as EMS, an enterprise messaging system is an enterprise-grade system that enables "program-to-program" messaging between applications and systems throughout an enterprise. Enterprise messaging is widely used today for integrating various disparate enterprise applications. It is a software interface that enables loosely coupled asynchronous data (messages) to be sent by one program and stored in a message queue until the receiving program is able to process it.

It's important to note that while the term messaging can be used to describe e-mail, fax, instant messages and other services where humans read and send the messages, in EMS terminology the word messaging is used to describe asynchronous messages that consists of requests, reports or events that are consumed by enterprise applications and not humans. These messages contain information that is needed to coordinate systems and track progress of the enterprise.

Enterprise messaging systems, which are language and platform-independent solutions, are also called messaging services, or more formally, messagingoriented middleware (MOM).

\section{Conclusions}

Integrated ERP computer systems incorporate high complexity, being designed to meet independent modular compartment requirements of an 
organization. For example, an ERP system has the following functional modules for an integrated information system for public administration institutions:

- Planning and tracking of production;

- Investment Management;

- Human Resources Management;

- Financial and Accounting Department;

- Project Management;

- Workflow;

- Monitoring-Control;

- Maintenance;

- Sales and Distribution;

- Management of Materials;

- Fixed Capital Management;

- Quality Management.

Integrated ERP computer systems have an architecture organized on three levels:

1)the presentation layer (work station);

2)the processing level of application type (business rules, logic and system functions, application programs, including transfer of data to server's data);

3)the data storage level in databases (database management and metadata).

There are quite a few manufacturers of integrated ERP systems. Thus, $60 \%$ of them are grouped under the acronym BOPS: Baan, Oracle, People Soft, SAP, JD Edwards. Most of ERP systems provide a flexible infrastructure, usually for business environment. Thus, ERP provide a number of features to their users: model.

$>$ Flexible infrastructure for business using Oracle and SAP companies as

Oracle uses Enterprise Service Bus (ESB), unified bus service as the basis for SOA (Services-Oriented Architecture).

$>$ ESB is a multi-protocol designed to separate the concepts of integration contained in applications of those contained in the business logic.

ESB is obtained by:

1)endpoints Virtualization (Endpoints) from resources to services;

2)transforming data in target- formats (target formats);

3)routing data through a variety of protocols;

4)laying out data as Web services wherever needed.

$>$ Standards used for the design of ERP systems: XP, XSLT, SOAP, JMS, JCA Hot: J2EE, JMS, Database etc.

BPEL (Business Process Execution) is an XML-based mark-up language for composing a set of discrete Web services in a process flow end-to-end.

$>$ BPEL provides a strong support for asynchronous interactions, parallel processing and exception management. 


\section{REFERENCES}

Gherasim, Z.; Andronie, M.; Popescu-Bodorin, N. (2004), Informatică managerială, Editura Fundației România de Mâine, Bucureşti.

Houston, N., ERP Implementation Strategies - A Guide to ERP Implementation Methodology, http:// blog.softwareadvice.com/articles/manufacturing/erpimplementation-strategies-1031101/, 11.03.2012.

Leovaridis, Cristina (2013), Aspects of innovation within Romania Entreprises, compared to other European countries, Annals of Spiru Haret University, vol. 4(13), issue 1, p. 21-32.

Maxim, Cornelia (2014), Analiza datelor şi gestiunea informatică a resurselor umane, Editura Tritonic.

Payne, A., Frow, Pennie (2013), Strategic customer management, Cambridge Universty Press.

Thomas, Frambach, Simon, Hoeg (2011), Floorplan Manager for Web Dynpro $A B A P$, first edition, Galileo Press.

Trană, D.M, Trană, R.D. (2008), Informatica pentru economiști, vol. 3 - Sisteme informatice de gestiune, Editura Sitech, Craiova. 